\title{
PC workstations in crystallography
}

\author{
Arno Pähler, Yuko Kojima and Takao Matsuzaki \\ (Mitsubishi Chemical Corporation, Yokohama Research Center)
}

With the advent of the Intel Pentium CPU a new class of workstations for scientific use have become available. They differ from other workstations mainly in one point: they are 5 to 10 times cheaper, while offering equal or higher performance. The priceperformance advantage of these personal workstations is a result of the mass-market nature of PCs, as opposed to the low volume sales of would-be real workstations. With $90 \mathrm{MHz}$ Pentium CPUs they are about as fast as IBM RS/6000-550 or Silicon Graphics Indigo2 and 1.2 to 1.5 times faster than SUN Sparc 10/50. A typical, real life comparison - based on manufacturer's offers and market prices - might look like the following example:

- SGI Indigo 2 with 128 MB memory/2 GB disk - 11,600,000 Yen

- Pentium WS with 128 MB memory/10 GB disk - 1,600,000 Yen

Both machines in this comparison have roughly equal performance. An illustration of the performance using the refinement program PROLSQ is shown in the table below. The last line shows the possibility for parallel computing using co-processor boards.

\begin{tabular}{|l||r|r||l||r|r|}
\hline System or CPU & Time & Ratio & System or CPU & Time & Ratio \\
\hline \hline VAX 11/780 & 1420.7 & 1.0 & i860-50MHz & 36.0 & 39.5 \\
\hline $80486 \mathrm{DX}-50 \mathrm{MHz}$ & 151.2 & 9.4 & IBM RS/6000-550 & 28.4 & 50.0 \\
\hline VaxStation 4000/60 & 145.6 & 9.8 & IBM RS/6000-580 & 18.4 & 77.2 \\
\hline SGI Crimson & 37.3 & 38.1 & Fujitsu VP-50 & 13.2 & 107.6 \\
\hline Pentium-90MHz & 36.6 & 38.8 & IBM RS/6000-590 & 12.0 & 118.4 \\
\hline \hline i860-25MHz & 53.6 & 26.5 & 4 xi860-25MHz & 19.8 & 71.6 \\
\hline
\end{tabular}

Pentium-based workstations can be run using linux, a free UNIX implementation. All the source code for Iinux is available and the available software is more complete than standard commercial UNIX implementations.

We have successfully ported a variety of crystallographic programs to be used on Pentium workstations running linux. These include:

\begin{tabular}{|c|l|}
\hline powder diffraction & RIETAN \\
small molecules & MULTAN 80 and 87, SHELXS-86, SHELXL93, ORTEP \\
protein structures & PROLSQ, FRODO, XTALVIEW, PROTEIN, CCP4,XPLOR \\
\hline
\end{tabular}

Porting is easiest for programs, which use standard language features of $\mathrm{C}$ or Fortran77. Programs, which use vendor-specific features, particularly DEC Fortran extensions, require more efforts. A particularly difficult example was W. Steigemann's PROTEIN system, as the VAX/VMS version was used as a starting point. Our Pentium workstations are networked using TCP/IP for communication and NFS for file sharing. Graphics is implemented through the $\mathrm{X} 11$ window system. 